\title{
Drug Preload
}

National Cancer Institute

\section{Source}

National Cancer Institute. Drug Preload. NCI Thesaurus. Code C152104.

The administration of an additional aliquot of an agent ahead of a standard dose, with the intent of raising the concentration of the agent in the compartment to which it is administered. 